\title{
Análise do atendimento nos serviços de saúde entre pessoas vivendo com HIV/AIDS
}

\section{Analysis of the care of health services between people living with HIV/AIDS}

\author{
Hévila Nascimento Gomes ${ }^{1}$ (D), Raimunda Hermelinda Maia Macena ${ }^{2}$ (D) , Gisele Maria Melo Soares Arruda ${ }^{2}$ (D), Ana Karine \\ Castelo Branco de Paula Gomes ${ }^{4}$ (i) \\ 1. Residente em Infectologia pela Escola de Saúde Pública do Ceará (ESP/CE), Fortaleza, CE, Brasil. 2. Docente do Programa de Pós-graduação em Saúde \\ Coletiva da Universidade Federal do Ceará (UFC), Fortaleza, CE, Brasil. 3 Discente do Programa de Pós-graduação em Saúde Coletiva da Universidade \\ Federal do Ceará (UFC), Fortaleza, CE, Brasil. 4. Discente do Programa de Pós-graduação em Gestão em Saúde pela Universidades Estadual do Ceará (UECE), \\ Fortaleza, CE, Brasil.
}

\section{Resumo}

Objetivo: analisar o atendimento dos serviços de saúde prestados às pessoas que vivem com HIV/AIDS, buscando identificar atitudes de discriminação e preconceito vivenciadas na perspectiva dos usuários no município de Fortaleza/CE. Método: estudo seccional e exploratório, com abordagem quantitativa, realizada no período de março a junho/2018, com 65 indivíduos que frequentam a unidade da Rede Nacional de pessoas que vivem com HIV/AIDS (RNP) na cidade de Fortaleza-CE. As atitudes de discriminação foram mensuradas por meio da escala de Allport. Os dados foram digitados pelo software Excel for Windows ${ }^{\circledR} 2010$ e, a seguir, exportados e analisados pelo programa STATA/SE® 11.2. Resultados: 20\% dos indivíduos afirmaram sofrer algum tipo de discriminação em locais de saúde por viverem com HIV/AIDS. Entretanto, 67,7\% informaram já terem sido vitimas de algum tipo de preconceito por causa da doença, desses $50 \%$ foram vítimas de piadas e apenas $6 \%$ de ataques físicos. Conclusão: ainda há muito a melhorar para uma efetiva garantia do acesso e humanização do atendimento às pessoas vivendo com HIV/AIDS.

Palavras-chave: Serviços de Saúde. HIV. SIDA. Estigma Social. Preconceito.

\begin{abstract}
Objective: to analyze the care provided to people living with HIV/AIDS, seeking to identify discrimination and prejudicial attitudes experienced by the users' perspective, in the city of Fortaleza. Method: this is a cross-sectional and exploratory field survey, with a quantitative approach, which was carried out between March and June 2018, with 65 individuals who attending the unit of the National Network of People Living with HIV/AIDS (RNP), located in the municipality of Fortaleza-Ceará. Discriminative attitudes were measured by the Allport Scale. The data was entered by the software Excel for Windows ${ }^{\circledR}$ 2010 and then was exported and analyzed by the STATA/SE® 11.2 program. Results: $20 \%$ of individuals reported having suffered some kind type of discrimination in health places due to the fact that they have HIV/AIDS. However, $67.7 \%$ reported that they had already suffered some kind of prejudice because of the disease, and that $50 \%$ were victims of jokes and only $6 \%$ of physical attacks. Conclusion: there is still much to improve for an effective guarantee of access and humanization of care for people living with HIV/AIDS.
\end{abstract}

Key words: Health Services. HIV. AIDS. Social Stigma. Preconception..

\section{INTRODUÇÃO}

A infecção pelo vírus HIV e a AIDS (do inglês Acquired Immune Deficiency Syndrome) têm feito muitas vítimas desde seu surgimento, e essas têm convivido com o estigma e a discriminação, acarretando inúmeras injustiças sociais ${ }^{1}$. $O$ fato de a síndrome ter afetado inicialmente os homossexuais e, com o passar do tempo, se disseminado, privilegiadamente, entre as profissionais do sexo, os usuários de droga e os moradores de rua (contextos de maior vulnerabilidade social e individual ${ }^{2}$ ), tornou a doença estigmatizada ${ }^{3,4}$.

A infeção por HIV/AIDS trouxe ao mundo não somente mais uma doença considerada incurável, que ainda causa medo e pânico na população, mas, principalmente, uma rediscussão de conceitos, preconceitos e comportamentos individuais e coletivos $^{3,4}$.
A epidemia da HIV/AIDS continua a ser um dos grandes desafios para a saúde global. Com base no Boletim Epidemiológico HIV/AIDS de 2018, do Sistema de Informação de Agravos de Notificação (SINAN), foram notificados, de 2007 até junho de 2018, 247.795 novos casos de infecção pelo Vírus da Imunodeficiência Humana (HIV) no Brasil. A taxa de detecção, em 2017, foi de 18,3/100.000 habitantes. Já a taxa de mortalidade, no mesmo ano, é de 4,8/100.000 habitantes ${ }^{5}$. No Ceará, foram notificados 20.226 casos de AIDS e 9.162 casos de HIV entre os anos de 1983 a 2018, com uma tendência de crescimento no período de 1996 a 2012. Somente em 2012, a incidência no sexo feminino foi duas vezes maior que em 1997, ano em que sucedeu a introdução da terapia antirretroviral no país ${ }^{6}$. A cidade de Fortaleza ocupou a 6a posição na detecção de casos entre as capitais da Região Nordeste, registrando mais da 
metade do total de casos do estado7.

Com o avanço da doença, algumas medidas foram tomadas pelo Ministério da Saúde para dar respostas à epidemia de HIV/ AIDS. Na área da assistência, foram criados serviços específicos como Hospitais Dia (HD), Serviços de Assistência Especializada (SAES), Centros de Testagens e Aconselhamentos (CTAS), e ainda a modalidade de Atendimento Domiciliar Terapêutico (ADTS), todos com a finalidade de proporcionar atendimento alternativo às formas tradicionais de tratamento ${ }^{4}$. Além disso, estratégias de diagnóstico precoce têm sido implementadas nos serviços de primeiro contato, viabilizando a antecipação e a descentralização do cuidado e, ao mesmo tempo, aproximando todos os profissionais da prevenção, do diagnóstico e do cuidado às pessoas vivendo com HIV/AIDS ${ }^{8,9}$. Outrossim, é relevante elencar a adoção de novas tecnologias, apesar dos desafios, para a ampliação das possibilidades de combate à epidemia de HIV por meio da profilaxia pré-exposição (PrEP) e da profilaxia pós-infecção $(P E P)^{10}$.

Entretanto, apesar de existir uma rede de atendimento especializada, as pessoas vivendo com HIV/AIDS ainda sofrem preconceitos e discriminação em toda a rede de atenção à saúde, seja no Sistema Único de Saúde (SUS), seja na assistência privada. Apesar de ser uma atitude antiética que contradiz os princípios do SUS, em geral, há uma dificuldade em se encontrar atendimento quando eles revelam a situação da doença aos profissionais de saúde ${ }^{11}$; ou mesmo quando acessam os serviços, diversas situações podem ocorrer, como o tratamento diferencial, a revelação da condição sorológica para outras pessoas, a isolação física não justificada biologicamente e o aconselhamento de intervenções que não seriam indicadas a outros pacientes, como o aborto e a esterilização. Há, ainda, a adoção de medidas diferenciadas de biossegurança, uma forma de discriminação bastante peculiar e praticada por muitos profissionais de saúde ${ }^{12,13}$

É incontestável que a epidemia da AIDS trouxe uma demanda diferenciada para os profissionais da saúde, pois, para proporcionar um cuidado humanizado, precisam lidar não somente com a assistência, mas também com o trato ao ser humano em suas questões mais íntimas ${ }^{4}$, bem como precisam acessar suas próprias questões pessoais, como o medo de infecção, os estigmas e os preconceitos atrelados à doença, que, tradicionalmente, esteve relacionada às drogas e às práticas sexuais questionáveis pela sociedade; a insegurança em lidar com uma notícia que pode gerar sofrimento; a exclusão social da pessoa atendida e angústias relacionadas à finitude da vida ${ }^{14,15}$. De acordo com Lelis et $a^{16}$, as principais formas de comportamento preconceituoso por parte de profissionais da saúde são a recusa de atendimento, as medidas de biossegurança exacerbadas, a falta de sigilo e até o isolamento físico da pessoa.

Contudo, a Associação Médica Americana (AMA) e outras associações de profissões da área da saúde determinaram que os profissionais por elas representados têm a obrigação ética de administrar cuidados a esses pacientes, desde que suas necessidades se enquadrem em suas áreas de competência ${ }^{17}$. Acrescente-se que, em todos os Códigos de Ética dos profissionais de saúde, existem imposições legais que condenam práticas discriminatórias no exercício profissional. Além disso, a Constituição Federal diz que todos os cidadãos brasileiros, sem exceções, têm garantido o direito à assistência à saúde de forma universal e igualitária ${ }^{18}$.

Diante disso, este estudo analisou o atendimento dos serviços de saúde prestados às pessoas que vivem com HIV/AIDS, buscando identificar atitudes de discriminação e preconceito vivenciadas na perspectiva dos usuários acompanhados pela Rede Nacional de Pessoas Vivendo com HIV/AIDS (RNP) na cidade de Fortaleza, Ceará

\section{MÉTODOS}

Estudo seccional e exploratório, com abordagem quantitativa, realizado no período de março a junho de 2018, após a aprovação do Comitê de Ética em Pesquisa do Hospital São José de Doenças Infeciosas, com o protocolo número 2568215.

Foram convidadas para participar da pesquisa, por conveniência, as pessoas vivendo com HIV/AIDS que frequentavam a unidade da Rede Nacional de pessoas vivendo com HIV/AIDS (RNP) e estavam presentes no período de dois meses da coleta de dados no turno da manhã, posto que este é o turno em que acontecem as reuniões e entregas de cestas básicas. A RNP, localizada no município de Fortaleza-Ceará, é uma organização nacional de pessoas vivendo com HIV/AIDS, sem vínculo políticopartidário e religioso, que atua na promoção do fortalecimento das pessoas vivendo com HIV/AIDS, independente de gênero, orientação sexual, credo, raça/cor ou etnia e nacionalidade19. Dos usuários mensais da RNP $(n=100), 80$ foram acessados e convidados a participar da coleta de dados, 15 se recusaram a participar, por medo de interferir na conquista do benefício, totalizando a amostra final em 65 indivíduos de ambos os sexos (perda $=15 \%$ ).

Os dados foram coletados por meio de questionário com duas questões descritivas e 23 objetivas, composto por dois blocos: um contendo o perfil sócio econômico (sexo, idade, cor da pele, estado civil, número de filhos, religião, escolaridade, tipo de moradia, situação ocupacional, renda, e benefícios sociais) e o outro o histórico da análise do atendimento (informações sobre diagnóstico e acompanhamento em serviços de saúde, incluindo a vivência de situações de preconceito e discriminação). Foi utilizada a Escala de Allport $^{20}$, reconhecida como método adequado para medir o preconceito em uma sociedade. A escala classifica o tipo de preconceito sofrido de acordo com os cinco itens abaixo:

- Antilocução - foi alvo de piadas sofridas por um grupo de pessoas.

- Esquiva - grupo de pessoas que evitaram contato.

- Discriminação - grupo tentando prejudicar, negando oportunidades, serviços.

- Ataque físico - ataques violentos contra indivíduos e grupos. 
- Extermínio - grupo majoritário busca a exterminação do grupo minoritário.

Os participantes foram convidados a assinalar quais os tipos de preconceito que eles já haviam sofrido.

Os dados foram digitados por meio do software Excel for Windows $^{\circledR} 2010$ e, posteriormente, exportados e analisados pelo programa STATA $/ \mathrm{SE}^{\circledR}$ 11.2. A análise descritiva concentrouse nas frequências, e estas foram expostas por meio de tabelas.

Os testes estatísticos não revelaram nenhuma associação importante.

\section{RESULTADOS}

A amostra foi composta por $53,8 \%$ de homens, com faixa etária entre 40 a 49 anos (52,3\%), com idade mínima de 25 anos, máxima de 73 anos e média de 44 anos, desvio-padrão $\pm 9,3$ anos e mediana de 45 anos; $60 \%$ se declararam pardos, 21,5\% brancos; $49,2 \%$ não têm instrução ou possuem o ensino fundamental incompleto, e apenas $9,2 \%$ têm o ensino superior completo ou incompleto; $53,8 \%$ são católicos e $30,8 \%$ evangélicos. Entre os indivíduos estudados, $64,6 \%$ são solteiros; $61,5 \%$ têm filhos; $60 \%$ vivem em casa própria, e $30,8 \%$ em casa alugada (TABELA 01).

Tabela 1. Caracterização demográfica da amostra. Fortaleza/CE, 2018

\begin{tabular}{lrr}
\hline Variável & N & (\%) \\
\hline Sexo & & \\
Masculino & 35 & 53,8 \\
Feminino & 30 & 46,2 \\
Faixa etária (anos) & & \\
$20-29$ & 3 & 4,6 \\
$30-39$ & 14 & 21,5 \\
$40-49$ & 34 & 52,3 \\
$50-59$ & 9 & 13,8 \\
$60-69$ & 4 & 6,2 \\
$>70$ & 1 & 1,5 \\
Raça & & \\
Branca & 14 & 21,5 \\
Parda & 39 & 60,0 \\
Preta & 9 & 13,8 \\
Outra & 3 & 4,5 \\
Escolaridade & & \\
Sem instrução/ Fundamental incompleto & 32 & 49,2 \\
Fundamental completo/ Médio incompleto & 11 & 16,9 \\
Médio completo & 16 & 24,6 \\
Superior incompleto/ completo & 6 & 9,2 \\
Religião (n=63) & & \\
\hline & &
\end{tabular}

\begin{tabular}{lrr}
\hline Variável & N & (\%) \\
\hline Católica & 35 & 53,8 \\
Evangélica & 20 & 30,8 \\
Outros & 4 & 6,2 \\
Não tem & 4 & 6,2 \\
NSNQR & 2 & 3,1 \\
Estado civil & & \\
Solteiro & 42 & 64,6 \\
Casado & 10 & 15,4 \\
Separado & 8 & 12,3 \\
Outros & 5 & 7,7 \\
Possui filhos & 40 & 61,5 \\
Tipo de moradia & & \\
Própria & 39 & 60,0 \\
Alugada & 20 & 30,8 \\
Cedida & 5 & 7,7 \\
Invadida & 1 & 1,5 \\
\hline
\end{tabular}

Fonte: Elaborado pelo autor (2018)

A minoria da amostra trabalha (16,9\%); 44,6\% recebem benefícios do governo, e $13,9 \%$ recebem pensão e/ou aposentadoria. Entre os programas sociais/ benefícios do governo, 26,2\% recebem o Bolsa Família. Quase metade dos indivíduos da amostra informou ser ele a principal fonte de renda da família (41,5\%). A renda individual é baixa: $38,5 \%$ recebem 1 salário-mínimo; 32,3\%, menos de 1 salário-mínimo e $27,7 \%$ não possuem qualquer tipo de renda (TABELA 02).

Tabela 2. Caracterização socioeconômica da amostra. Fortaleza/ CE, 2018.

\begin{tabular}{lrr}
\hline Variável & N & (\%) \\
\hline Trabalha & 11 & 16,9 \\
Origem da renda & & \\
Não recebe renda & 18 & 27,7 \\
Salário & 6 & 9,2 \\
Pensão & 2 & 3,1 \\
Aposentadoria & 6 & 9,2 \\
Aposentadoria e pensão & 1 & 1,6 \\
Benefício & 29 & 44,6 \\
Outros & 3 & 4,6 \\
Principal fonte de renda da família & 27 & 41,5 \\
Renda individual & & \\
Sem renda & 18 & 27,7 \\
Menos de 1 salário-mínimo & 21 & 32,3 \\
1 salário mínimo & 25 & 38,5 \\
Mais de 1 salário-mínimo & 1 & 1,5 \\
\hline
\end{tabular}

Fonte: Elaborado pelo autor (2018) 
Entre os indivíduos estudados, $73,86 \%$ realizaram o teste de HIV em hospital público ou durante o pré-natal; 16,92\% em um centro de saúde e 6,15\% em hospital/laboratório particular. A primeira consulta com médico, após o resultado do teste, foi realizada em menos de 1 mês $(47,7 \%)$ ou de 1 a 3 meses $(44,6 \%)$. A maioria da amostra revelou o resultado do teste para alguém (76,9\%).

Entre os sujeitos do estudo, 20,0\% informam que são tratados, de maneira diferente, em algum serviço por ser portador do HIV, sendo esse evento mais frequente na rede terciária $(7,69 \%)$ e na primária (7,69\%). Entre os profissionais que trataram as pessoas vivendo com HIV/AIDS - PVHA de alguma forma diferente, o médico e o enfermeiro são os que mais atuaram com diferença no atendimento $(4,61 \%$ cada $)$. Apenas $15,4 \%$ da amostra relatam ter ficado com algum problema físico (problemas no corpo) e/ou psicológico (problemas emocionais) por causa desse ato. Todavia, se levarmos em consideração aqueles que foram tratados diferentes, $76,9 \%$ deles relatam dano físico e psicológico como consequência dessa discriminação.

Um pouco mais da metade da amostra $(50,77 \%)$ informou já ter sofrido antilocução, 44,6\% discriminação, 43,07\% esquiva, $6,15 \%$ ataque físico e 6,15\% extermínio. Quase $1 / 4$ da amostra $(21,5 \%)$ relata ter sofrido apenas 1 tipo de ação preconceituosa; $20 \%$ informaram três ações; e 18,5\% assinalaram duas ações. A amostra avaliou também o atendimento nos serviços de saúde, em que $75,3 \%$ avaliaram como bom ou excelente, e 9,3\%, como ruim ou péssimo (TABELA 03).

Tabela 3. Análise dos serviços de saúde (teste, consulta, resultado do teste, tratamento e avaliação). Fortaleza/CE, 2018

\begin{tabular}{|c|c|c|}
\hline Variável & $\mathbf{N}$ & $(\%)$ \\
\hline \multicolumn{3}{|l|}{ Sobre a testagem para HIV } \\
\hline \multicolumn{3}{|l|}{ Local de realização do teste } \\
\hline Hospital público e pré-natal & 48 & 73,86 \\
\hline Hospital e laboratório particular & 4 & 6,15 \\
\hline Banco de sangue & 2 & 3,07 \\
\hline Centro de saúde & 11 & 16,92 \\
\hline \multicolumn{3}{|l|}{ 1‥ Consulta após resultado do teste $(n=64)$} \\
\hline Menos de 1 mês & 31 & 47,7 \\
\hline 1 a 3 meses & 29 & 44,6 \\
\hline 3 a 6 meses & 4 & 6,2 \\
\hline NSNQR & 1 & 1,5 \\
\hline Contou para alguém sobre resultado do teste & 50 & 76,9 \\
\hline Foi tratado diferente por ser portador do HIV & 13 & 20,0 \\
\hline \multicolumn{3}{|l|}{ Local em que foi tratado diferente } \\
\hline Atenção primária & 5 & 7,69 \\
\hline Atenção secundária & 2 & 3,08 \\
\hline Atenção terciária & 5 & 7,69 \\
\hline NSQRS & 1 & 1,54 \\
\hline \multicolumn{3}{|l|}{ Profissional que tratou diferente } \\
\hline Enfermeiro & 3 & 4,61 \\
\hline Técnico de enfermagem & 2 & 3,07 \\
\hline Assistente Social & 1 & 1,53 \\
\hline Médico & 3 & 4,61 \\
\hline Outros profissionais & 4 & 7,14 \\
\hline Dano físico/psicológico por ter sido tratado diferente & 10 & 15,4 \\
\hline \multicolumn{3}{|l|}{ Escala de Allport } \\
\hline Antilocução & 33 & 50,77 \\
\hline Discriminação & 29 & 44,60 \\
\hline Esquiva & 28 & 43,07 \\
\hline
\end{tabular}




\begin{tabular}{lrr}
\hline Variável & $\mathbf{N}$ & (\%) \\
\hline Ataque físico & 4 & 6,15 \\
Extermínio & 4 & 6,15 \\
Número de itens sofridos (Escala de Allport) & 21 & 32,3 \\
Nenhum item & 14 & 21,5 \\
1 item & 12 & 18,5 \\
2 itens & 13 & 20,0 \\
3 itens & 4 & 6,2 \\
4 itens & 1 & 1,5 \\
5 itens & & 15 \\
Avaliação do atendimento nos serviços de saúde & 34 \\
Excelente & 10 & 23,1 \\
Bom & 2 & 52,2 \\
Regular & 4 & 15,4 \\
Ruim & 4 & 3,1 \\
Péssimo & 6,2 \\
\hline
\end{tabular}

Fonte: Elaborado pelo autor (2018)

\section{DISCUSSÃO}

Neste estudo, diferentemente de outros pesquisados, a maioria é do sexo masculino. No entanto, o atual perfil da doença tem demonstrado que todos estão vulneráveis a contrair a doença, sendo indiferente ser do sexo masculino ou feminino ${ }^{1,3}$. A epidemia de HIV/AIDS vem sofrendo diversas modificações em seu perfil ao longo do tempo, entre as quais, os fenômenos de feminização, heterossexualização, pauperização e envelhecimento, ou seja, os padrões de vulneráveis ao vírus têm-se modificado, e não há mais a antiga estereotipação dos sujeitos vulneráveis ${ }^{2,21,22}$.

Em relação à idade dos participantes, a média é de 44 anos, sendo predominante a faixa etária entre 40 e 49 anos (52\%). Os resultados diferem de alguns estudos que mostram que, nos últimos 10 anos (2007 a 2017), as maiores taxas de detecção de AIDS foram observadas entre aqueles com idade de 15 a $29 \operatorname{anos}^{23}$. Quanto à religião, a maioria dos entrevistados é adepta ao catolicismo. Segundo pesquisa realizada por Garcia e Sousa ${ }^{24}$, a orientação religiosa parece não exercer interferência direta nas práticas e nos comportamentos sexuais.

Analisando o estado civil, a maioria declara ser solteira, e pode haver uma variação de parceiros que, consequentemente, pode contribuir para a propagação de doenças sexualmente transmissíveis, como a AIDS. Isso representa um maior desafio no que diz respeito à prevenção e à profilaxia por parte dos serviços de saúde ${ }^{10}$. A maioria dos entrevistados não possui filhos, o que inviabiliza uma maior investigação sobre ocorrência, ou não, de transmissão vertical do vírus. Esse tipo de contaminação tem sido responsável por cerca de $90 \%$ dos casos notificados de AIDS em menores de 13 anos $^{25}$.

Em relação à cor/etnia, os achados deste estudo se assemelham aos divulgados pelo Instituto de Pesquisa e Estratégia Econômica do Ceará - IPECE, o qual mostra que 71,1\% dos cearenses se declaram pardos ou pretos ${ }^{26}$. Os dados vão ao encontro de uma análise descritiva da base de dados do Programa Nacional-DST/AIDS, que sugere ter ocorrido uma reversão de tendência de pauperização da epidemia de HIV/AIDS e que a suposta elevação da "população negra" resulta de um aumento somente entre os pardos, mas não entre os pretos. Esse último dado é de particular relevância, considerando que as campanhas de AIDS/HIV disseminadas pelo governo brasileiro estão, particularmente, voltadas para as pessoas de fenótipo mais "escuro"27.

O nível de escolaridade dos usuários é baixo, o que pode estar associado à falta de acesso às informações sobre a doença e às formas de prevenção ${ }^{3}$. Evidencia-se que, quando a epidemia surgiu, ela se concentrava nas camadas mais abastadas da sociedade e com níveis de escolaridade mais elevados, mas, como mostram os dados, ela também tem atingindo as camadas menos favorecidas.

Os dados revelam que a maioria não trabalha, mesmo sendo pessoas em idade produtiva, o que nos leva a refletir se esses usuários podem estar sendo vítimas de discriminação por serem pessoas que vivem com HIV/AIDS. É importante enfatizar que negar trabalho ou demitir sem justa causa uma pessoa pelo fato de estar vivendo com HIV/AIDS constitui uma forma de preconceito e discriminação. Entre os direitos conquistados pela PVHAs, está a reintegração do trabalhador demitido por discriminação, além de condenação da empresa ao pagamento de indenização por danos morais e materiais ${ }^{3,4,11}$.

Identificou-se que a maioria dos entrevistados possui renda 
originária de benefício social de Benefício de Prestação Continuada (BPC), recebendo um salário mínimo. Com o aumento da infecção pelo HIV, e, consequentemente, da AIDS, nas camadas de baixa renda, o BPC tem sido uma alternativa de renda, visto que, a depender do nível de carga viral, muitos não têm condições de trabalhar ${ }^{3}$.

Vale salientar que a RNP também é uma rede de cunho social, o que acaba abrangendo um público menos favorecido que procura a entidade não só para fortalecer os seus direitos, mas também para receber cestas básicas e apoio psicológico. Assim, a pesquisa mostrou que o perfil do público atendido pela RNP possui baixa renda, fazendo que eles procurem o benefício BPC.

A pesquisa mostrou que, apesar da baixa renda, a maioria possui casa própria. Entretanto, vale destacar que, apesar de alguns avanços no que se refere à questão da moradia, como um maior acesso da população de baixa renda à casa própria, ainda existe um grande déficit habitacional em nosso país. Nesse sentido, há a necessidade de se compreender que a habitação é muito mais que um teto, é o espaço em que se vive, abarcando vários equipamentos, como esgotamento sanitário, transporte, escolas, serviços de saúde e outros.

Quanto ao atendimento dessas pessoas em locais de saúde, observa-se que há elevados índices de discriminação. Isso mostra que as atitudes discriminatórias que as pessoas com HIV/ AIDS vivenciam nos serviços de saúde trazem consequências negativas para o controle da epidemia, pois há uma diminuição pela busca precoce do diagnóstico, formando-se uma barreira crítica para as pessoas buscarem os cuidados da saúde ${ }^{28}$.

No Brasil, o Programa Nacional de IST e AIDS, juntamente com seus parceiros, atua em 390 municípios nos vinte e seis estados da federação e no Distrito Federal, os quais apresentam $80 \%$ do número de casos de AIDS no País. Os objetivos do programa são reduzir a incidência da infecção pelo HIV/AIDS e outras IST; ampliar o acesso e melhorar a qualidade do diagnóstico, tratamento e da assistência aos portadores do HIV/AIDS e outras IST; e fortalecer as instituições públicas e privadas responsáveis pelo controle das IST/HIV/AIDS ${ }^{29}$.

Ressalta-se que os entrevistados relataram que buscam por redes de atendimento especializado em HIV/AIDS, pois, segundo eles, seriam uma forma de minimizar o tratamento diferenciado por parte dos profissionais.

Percebe-se que o diagnóstico positivo ao HIV ainda é relacionado ao medo, ao isolamento social, à perda do emprego, ao afastamento da família e dos amigos. Esse receio diminui a busca pelo conhecimento da condição sorológica do indivíduo, favorece o silêncio acerca do seu diagnóstico e inibe a procura pelo acesso aos tratamentos disponíveis na rede de saúde ${ }^{30}$.

Contudo, a discriminação das pessoas que vivem com HIV/ AIDS por profissionais de saúde tem sido observada em vários estudos ${ }^{11,13,30}$, e isso acontece por alguns motivos; entre eles, destacam-se o medo da contaminação decorrente do pouco conhecimento sobre a transmissão do HIV, a associação da doença com comportamentos impróprios e imorais, além da falta de conscientização da existência do preconceito e suas consequências ${ }^{30}$.

Dentro da categoria "outros profissionais", o dentista foi o profissional mais citado como quem trata de maneira diferente as PVHA, o que vai ao encontro da pesquisa de Jovic-Vranes et al. $^{13}$, a qual avaliou o comportamento de profissionais de saúde em relação ao atendimento de pessoas com HIV. Neste estudo, observou-se que os cirurgiões-dentistas tinham mais receio de atender a essas pessoas do que os médicos e os outros profissionais da saúde, e que medidas adicionais de biossegurança eram adotadas quando atendiam a esses indivíduos.

Diante desses fatos, é importante que os profissionais de saúde estejam sempre trabalhando a Educação Permanente em Saúde (EPS), que traz como marco conceitual uma concepção de trabalho no SUS como aprendizagem cotidiana e comprometida com os coletivos. É necessário que, desde o começo da sua inserção em locais de trabalho, do estágio à formação, o profissional já tenha essa visão de atendimento humanizado aos pacientes, independentemente da condição de saúde ou de qualquer outro fator intrínseco a ele ${ }^{31}$.

Em relação à Escala de Allport, pode-se perceber que ainda há um forte preconceito em relação à teoria de que muitos portadores do vírus ainda sofrem com violência verbal. Vale ressaltar, também, que, apesar da eficácia das campanhas governamentais com o objetivo de esclarecimento sobre a síndrome e as formas de infecção, o conhecimento em si não é suficiente para mudar atitudes em relação às pessoas portadoras do vírus e às práticas preventivas.

Enfim, percebe-se que essa questão vai além da informação e exige mudanças de visão de mundo, de crenças e de aceitação do outro na sua singularidade e diversidade ${ }^{32}$. Acredita-se que ainda haja medo e falta de informação a respeito da doença, mesmo após vários investimentos e campanhas nesse sentido. Essas discussões objetivam compreender a importância desses elementos nos processos de saúde e adoecimento, como também na promoção da saúde ${ }^{21}$.

A limitação do trabalho repousa na elevada recusa das pessoas vivendo com HIV em responder ao questionário, por medo da perda do benefício financeiro governamental ou da modificação do tipo de atendimento nos serviços de saúde.

\section{CONCLUSÃO}

Pelo fato de a infecção por HIV/AIDS ser uma enfermidade crônica, ainda estigmatizada, as questões de natureza psicossocial são de grande relevância, somando-se aos desafios da esfera biomédica. A constituição de equipes interdisciplinares parece ser de fundamental importância para a qualificação da assistência prestada, facilitando uma abordagem adequada às dificuldades médicas e psicossociais de pessoas vivendo com HIV/AIDS. 
Mesmo assim, a pesquisa mostrou que, em geral, os pacientes consideram o atendimento de saúde como bom, apesar de haver alguns tratamentos não adequados.

Diante do exposto, considera-se que as políticas públicas, em âmbito nacional, devem continuar investindo em processos de descentralização, ampliando as tecnologias de diagnóstico, tratamento, prevenção e realização de estudos epidemiológicos periódicos em todos os níveis. É necessário expandir sua análise e interpretação para o nível local, objetivando uma aplicação mais efetiva dessas informações para representar parâmetros de avaliação das políticas públicas de prevenção e controle.
Ressalta-se que o presente estudo apresenta algumas limitações, como a opção metodológica e o recorte amostral, dado em que se abordou um segmento bastante específico da população. Não é possível produzir generalizações; todavia, acredita-se que o estudo contribui na análise de aspectos importantes quanto à percepção das pessoas vivendo com HIV/AIDS sobre discriminação e preconceito na assistência em saúde. Por se tratar de uma amostra com condições socioeconômicas mais desfavoráveis, os resultados aqui apresentados viabilizam uma discussão que evidencia a necessidade de humanização da assistência e a melhor formação dos profissionais dos serviços de saúde públicos do Ceará.

\section{REFERÊNCIAS}

1. Guimarães MDC al. Mortalidade por HIV/Aids no Brasil, 2000-2015: motivos para preocupação? Rev. bras.epidemiol. 2017; 20(suppl. 1): 182-190. doi: http://dx.doi.org/10.1590/1980-5497201700050015.

2. Ayres JRCM, Calazans GJ, Saletti Filho HC, França-Junior I. Risco, vulnerabilidade e práticas de prevenção e promoção da saúde. In: Campos GWS, Bonfim JRA, Minayo MCS, Akerman M, Drumond M Júnior, Carvalho YM. Tratado de Saúde Coletiva. São Paulo: Hucitec, 2009; p. 375-418.

3. Greco DB. Trinta anos de enfrentamento à epidemia da Aids no Brasil, 1985-2015. Ciênc. saúde coletiva. 2016; 21(5): 1553-1564. doi: http://dx.doi. org/10.1590/1413-81232015215.04402016.

4. Villarinho MV, Padilha MI, Berardinelli LMM, Borenstein MS, Meirelles BHS, Andrade SR. Políticas públicas de saúde face à epidemia da AIDS e a assistência às pessoas com a doença. Rev. bras. enferm. 2013 Mar.-Abr.; 66(2): 271-277. doi: http://dx.doi.org/10.1590/S0034-71672013000200018.

5. Ministério da Saúde (BR). Bol Epidemiol: aids e DST[Internet]. 2018 [acesso2019 Jul 14]; 49. Disponível em: http://www.aids.gov.br/pt-br/ pub/2018/boletim-epidemiologico-hivaids-2018.

6. Ceará. Secretaria de Saúde do Ceará. Boletim Epidemiológico. 2018 Nov 30 [acesso 2019 jul 14]. Disponível em: https://www.saude.ce.gov.br/download/ boletins/.

7. Szwarcwald $C L$, Souza $P R$ Júnior, Damacena $G N$, Junior $A B$, Kendall $C$. Analysis of data collected by RDS among sex workers in 10 Brazilian cities, 2009: estimation of the prevalence of HIV, variance, and design effect. J Acquir Immune Defic Syndr. 2011 Aug ;57(Suppl 3): S129-S135. doi:http;//dx.doi. org/10.1097/QAI.0b013e31821e9a36.

8. Araújo WJ, Quirino EMB, Pinho CM, Andrade MS. Perception of nurses who perform rapid tests in Health Centers. Rev Bras Enferm [Internet]. 2018; 71(Suppl 1):631-6. doi: http://dx.doi.org/10.1590/0034-7167-2017-0298.

9. Silva ITS, Valença CN, Silva RAR. Cartografia da implementação do teste rápido anti-HIV na Estratégia Saúde da Família: perspectiva de enfermeiros. Esc. Anna Nery [Internet]. 2017 Set [acesso 2019 Jul 05]; 21(4): e20170019. Disponível em: http://www.scielo.br/scielo.php?script=sci_ arttext\&pid=S141481452017000400222\&Ing=en.

10. Maksud I, Fernandes NM, Filgueiras SL. Tecnologias de Prevenção do HIV e desafios para os serviços de saúde. Rev. bras. epidemiol. 2015 Set.; 18(Suppl 1): 104-119. doi: http://dx.doi.org/10.1590/1809-4503201500050008.

11. Garbin CAS, Garbin AJI, Moimaz SAS, Carmo MP. Bioética e HIV/AIDS: discriminação no atendimento aos portadores. Rev. Bioét, 2009; 17 (3):511-22

12. Kermode M, Holmes W, Langkham B, Thomas MS, Gifford S. Hiv-related knowledge, attitudes \& risk perception amongst nurses, doctors \& other healthcare workers in rural india. Indian J Med Res. 2005 Sep; 122(3): 258-264
13. Jovic VA, Jankovic S, Vukovicd VB, Miljus D. Risk perception and attitudes towards HIV in Serbian health care workers. Occup Med. 2006 Jun; 56(4): 275 278. doi: 10.1093/occmed/kql019.

14. Monteiro JFA, Figueiredo MAC. Vivência profissional: subsídios à atuação em HIV/AIDS. Paidéia [Internet]. 2009 Abr [acesso 2019 Jul 04]; 19(42): 67-76. Disponível em: http://www.scielo.br/scielo.php?script=sci_arttext\&pid=S0103$863 \times 2009000100009$

15. Shima E, Nogueira MMCF, Nogueira ML A. The experience of infectologists faced with death and dying among their patients over the course of the AIDS epidemic in the city of São Paulo: qualitative study. Med. J [Internet]. 2010 [acesso 2019 Jul 04]; 128(2): 74-80. Disponível em: http://www.scielo.br/pdf/ spmj/v128n2/a06v1282.pdf.

16. Lelis RT, Garbin CAS, Garbin AJI, Soares GB. Vivendo com HIV/AIDS: estudo da ocorrência de discriminação nos serviços de saúde. Rev. Bras. Pesq. Saúde. 2012 Out-Dez; 14(4): 22-28.

17. Bryan CS. HIV/AIDS and bioethics: historical perspective, personal retrospective. Health Care Anal, 2012; 10(1): 5-18. doi: 10.1023/A:1015634125917.

18. Brasil. Constituição da República Federativa do Brasil. São Paulo: Revista dos Tribunais; 1999.

19. Rede Nacional de Pessoas Vivendo com HIV/AIDS. Carta de princípios da RNP+ Brasil. Brasília: RNPVHA; 2018 [acesso 2018 Jan 19]. Disponível em: http://www.rnpvha.org.br/carta-de-principios-da-rnp-brasil.html.

20. Allport GW. The nature of prejudice. Reading, Mass: Addison-Wesley; 1954.

21. Cassétte JB, Silva LC, Felício EEAA, Soares LA, Morais RA, Prado TS et al . HIV/aids em idosos: estigmas, trabalho e formação em saúde. Rev. bras. geriatr. gerontol. 2016; 19(5): 733-744. doi: https://dx.doi.org/10.1590/1809 98232016019.150123.

22. Leal AF, Knauth DR, Couto MT. A invisibilidade da heterossexualidade na prevenção do HIV/Aids entre homens. Rev. bras. epidemiol. 2015; 18 (Suppl 1): 143-155. doi: 10.1590/1809-4503201500050011.

23. Ministério da saúde (BR). Secretaria de Vigilãncia em Saúde. Boletim epidemiológico: AIDS e DST [Internet]. Brasília: Ministério da Saúde; 2017 [acesso 2017 Out 12]. Ano V, n. 01. Disponível em: <file://C:/Users/User/ Downloads/boletim_aids_internet.pdf $>$.

24. Garcia S, Souza FM. Vulnerabilidades ao HIV/aids no Contexto Brasileiro: iniquidades de gênero, raça e geração. Saúde Soc. 2010 Dez; 19(supl. 2): 9-20.

25. Moura EL, Praça N. Transmissão vertical do HIV: expectativas e ações da gestante soropositiva. Rev Latino-am Enfermagem [Internet]. 2006 [Acesso 2014 Jul 16]; 14: 405-413. Disponível em: http://www. file:///C:/Users/ / 
Downloads/2311-3315-1-PB.pdf.

26. Instituto de Pesquisa e Estratégia Econômica do Ceará. Síntese dos Indicadores Sociais do Ceará 2017[acesso 2018 Set 29]. Fortaleza: IPECE; 2018. Disponível em: https://www.ipece.ce.gov.br/wp-content/uploads/ sites/45/2019/01/Indicadores_Sociais_2017.pdf.

27. Fry PH, Monteiro S, Maio MC, Bastos FI, Santos RV. AIDS tem cor ou raça? Interpretação de dados e formulação de políticas de saúde no Brasil. Cad. Saúde Pública. 2007 Mar; 23(3): 497-523. doi: http://dx.doi.org/10.1590/S0102$311 \times 2007000300002$.

28.Li X, Lu H, Ma X, Sun Y, He X, Li C, et al. HIV/ AIDS-related stigmatizing and discriminatory attitudes and recent HIV testing among men who have sex with men in Beijing. AIDS Behav. 2012 Apr; 16(3):499-507. doi: 10.1007/s10461-0120161-x.
29. Garcia S, Koyama MAH. Estigma, discriminação e HIV/Aids no contexto brasileiro, 1998 e 2005. Rev. Saúde Pública. 2008; 42(Suppl 1): 72-83. doi: http://dx.doi.org/10.1590/S0034-89102008000800010.

30. Nyblade L, Stangl A, Weiss E, Ashburn K. Combating HIV stigma in health care settings: what works? J Int AIDS Soc 2009; 12:15. doi: 10.1186/1758-2652 12-15.

31. Vasconcelos DC, Coêlho AEL. Conhecimentos, atitudes e percepção de risco dos acadêmicos de farmácia frente a AIDS. Rev. Psicol. Saúde. 2013 Dez; 5(2): 109-117.

32. Meirelles BHS, Silva DMGV, Vieira FMA, Souza SS, Coelho IZ, Batista R. Percepções da qualidade de vida de pessoas com HIV/aids. Rev Rene. 2010; 11(3): 68-76. 